\title{
A Probabilistic Kernel Method for Human Mobility Prediction with Smartphones.
}

\author{
Trinh Minh Tri Do ${ }^{\mathrm{a}}$, Olivier Dousse ${ }^{\mathrm{b}}$, Markus Miettinen $^{\mathrm{b}}$, Daniel \\ Gatica-Perez $^{\mathrm{a}, \mathrm{c}}$ \\ ${ }^{a}$ Idiap Research Institute, Martigny, Switzerland \\ ${ }^{b}$ Nokia Research Center, Lausanne, Switzerland \\ ${ }^{c}$ EPFL, Lausanne, Switzerland
}

\begin{abstract}
Human mobility prediction is an important problem which has a large number of applications, especially in context-aware services. This paper presents a study on location prediction using smartphone data, in which we address modeling and application aspects. Building personalized location prediction models from smartphone data remains a technical challenge due to data sparsity, which comes from the complexity of human behavior and the typically limited amount of data available for individual users. To address this problem, we propose an approach based on kernel density estimation, a popular smoothing technique for sparse data. Our approach contributes to existing work in two ways. First, our proposed model can estimate the probability that a user will be at a given location at a specific time in the future, by using both spatial and temporal information via multiple kernel functions. Second, we also show how our probabilistic framework extends to a more practical task of location prediction for a time window in the future. Our approach is validated on an everyday life location datasets consisting of 133 smartphone users. Our method reaches an accuracy of $84 \%$ for the next hour, and an accuracy of $77 \%$ for the next three hours.
\end{abstract}

Keywords: smartphone, human mobility, prediction, personalized service

Email addresses: do@idiap.ch (Trinh Minh Tri Do), olivier.dousse@here.com (Olivier Dousse), markus.miettinen@trust.cased.de (Markus Miettinen), gatica@idiap.ch (Daniel Gatica-Perez)

Preprint submitted to Pervasive and Mobile Computing

August 18, 2014 


\section{Introduction}

The advances in mobile sensing and computing have enabled the integration of machine learning into personal mobile devices. In particular, smartphones emerge as all-purpose devices with personalized services, where the personalization is based on what the smartphone knows about the user. Smartphones can unobtrusively collect data about where a user goes and what they do, and build a detailed understanding of the user. First, the recorded data can be used to characterize multiple aspects of the user including demographic information [1, 2] or personality [3. Second, clustering methods can be applied to extract recurrent user contexts such as commonly visited places [4, providing a high level representation of context (instead of raw measurements). Finally, along with extracting and organizing information from the past, the phone can also learn a behavior model that can predict future activities and venues.

Location prediction can benefit mobile applications and services by letting the applications adapt to possible movements of the user. This can help a mobile device, for instance, to adapt its user interface based on the anticipated locations that the user will visit during the course of a day. As one example, it can prefetch and display relevant information related to the predicted target locations. Note that, for such scenarios, personalization is key as the interest does not lie in predicting the places that people are likely to visit, but rather in anticipating the movements of a single user. Also, since location traces of users are highly privacy-sensitive, it is not desirable to rely on a solution that requires location traces to be aggregated to a central data storage. Therefore, the prediction method has to be such that it relies only on the context history of a specific user for whom the prediction will be made.

Previous studies on mobility prediction have usually focused on predicting the next place where a user goes [5. However, in practice, the prediction capability needs to go beyond the anticipated next place of the user, and instead provide predictions for different look-ahead periods. This is because applica- 
tions might want to provide different kinds of information, depending on how imminent the user's visit to a location will be. For example, an application may want to prefetch traffic information for the route to a place that the user is predicted to visit during the next few hours, but for visits farther away in the future ( during the next 24 hours) other information like a weather forecast

35 for the target location might be more appropriate. With these requirements in mind, we develop a flexible prediction method which can predict user location for a given timestamp or for different look-ahead time windows.

The mobility prediction problem can be formalized as a contextual prediction problem where the future movements are assumed to depend only on the user context, which is characterized by space and time in this paper. The assumption is based on the repetitive nature of human mobility: similar contexts might imply similar movements in the future. For example, from the mobility traces of a given user, one might observe that if he is at a given train station around 8:00 AM on Monday then he will likely be at work around 8:25 AM.

45 Under a probabilistic framework, the location prediction task consists on estimating the conditional distribution over the set of future location candidates for a given specific context, based on mobility history. This can be modeled by representing the user context as a combination of discrete states (e.g., at place $\mathrm{X}$ at hour $\mathrm{Y}$ on day $\mathrm{Z}$ ), and so the conditional distribution is proportional to 50 the counts of possible outputs for the considered context. This approach, however, suffers from a major issue with discretization: the relationship between states are lost. For example, if we discretize the time of day into 24 time slots by hour, then 7:59 AM and 8:00 AM belong to two completely different time slots, while they are actually very close. We resolve this problem by using kernel density estimation (KDE), a non-parametric approach, for the estimation of the conditional probabilities. The idea is to use kernel functions to measure the similarity between the current context and data points in the location history. Data points with the highest similarity scores will have significant impact on the outputs. This approach is advantageous for dealing with sparse data, which happens when the amount of data is limited or when the user is in an infrequent 
context.

Our paper makes two major contributions. First, we propose a non-parametric approach for location prediction based on kernel density estimation, for which we introduced several kernels to capture different aspects of spatio-temporal context. Our fully probabilistic framework can make predictions for a specific time or for a look-ahead time-window without any heuristics. Second, we present a thorough application-oriented study of location prediction, which considers the look-ahead time interval as a key aspect. Our analysis is conducted on a real life datasets with state-of-the-art spatial resolution and longitudinal recording period. Our experimental results show how the prediction performance is affected by various factors such as the time of the day or the look-ahead time window.

The paper is organized as follows. The next section discusses related work on mobility prediction in the context of mobile computing and compare our contributions with respect to the existing literature. Section 3 presents our prediction frameworks, with formal descriptions of the data representation and the prediction task, that is to predict user location at a given time in the future. Our analysis starts in Section 4, which introduces the location dataset. We report baseline results in Section 5 The results reveal the contexts for which the baseline performance is low, and motivates our proposed KDE approach 80 presented in Section 6, a probabilistic model which uses spatio-temporal context. While the proposed method improves the accuracy on difficult settings such as large look-ahead time, we also found situations in which a simple baseline works best. Section 7 thus presents our final solution, which is a combination of the proposed model and a probabilistic version of the baseline method. In Section 8 . we generalize the framework from predictions for a specific time, to predictions for a time window, reporting experimental results with information retrieval measures appropriate for this new task. Finally, Section 9 provides concluding remarks. 


\section{Related Work}

90 development of location tracking techniques [6, 7]. Song et al. 8 presented a study on predictability of human mobility by analyzing the entropy of location traces. The analysis of entropy shows that the limit of predictability is around $93 \%$ for hourly sequences of GSM cell IDs, where the average size of a cell's ing predictability of discrete time series coming from several sources including GSM, WLAN, Bluetooth, and accelerometer. Recently, Lin et al. [10] extended the original work by studying the effect of spatio-temporal scales on predictability, showing that predictability increases with spatial scale and decreases with temporal scale.

Several prediction methods have been proposed for human mobility in different contexts (i.e., using different devices and sensors) and with different definitions of the prediction task. Some notable works are listed in Table 1. In transportation, Krumm et al. [12] consider the problem of inferring the destination based on partial paths which could be applied in navigation assistance systems. For example, context-aware trip recommendations can be produced by combining user specific needs (e.g., finding a gas station) with the inferred primary destination [19]. At a higher level, the prediction task is to infer people's movement among places such as "If the user is currently at home, which actly the prediction task is defined. In an early analysis with GPS traces of seven users over several months [5], Ashbrook et al. proposed to extract significant places and represent location traces as strings, then use Markov models to predict the next place that a user will visit. Song et al. 11] investigated various prediction methods on symbolic location traces collected with WiFi access points of a university campus. A few works have attempted to improve the prediction performance by exploiting other smartphone data beside location, such as call logs, Bluetooth, and application usage [15]. However it is still challeng- 
Table 1: Notable works on human mobility prediction

\begin{tabular}{|c|c|c|c|c|}
\hline Study & Prediction task & Data type & Data collection devices & $\begin{array}{l}\text { Population and } \\
\text { duration }\end{array}$ \\
\hline Markov models 5] & next location & GPS & GPS device & 7 users, $3-7$ months \\
\hline $\begin{array}{l}\text { String based prediction } \\
11\end{array}$ & next location & $\mathrm{WiFi}$ & $\begin{array}{l}543 \mathrm{WiFi} \text { APs within a } \\
\text { campus }\end{array}$ & 6000 users, 2 years \\
\hline Predestination 12 & trip destination & GPS & GPS-equipped car & $\begin{array}{l}169 \text { subjects, two } \\
\text { weeks }\end{array}$ \\
\hline $\begin{array}{l}\text { Present/absent probability } \\
13\end{array}$ & $\begin{array}{l}\text { home/away } \\
\text { prediction }\end{array}$ & GPS & smartphones & 34 users, 8 weeks \\
\hline NextPlace [14] & $\begin{array}{ll}\text { future } & \text { loca- } \\
\text { tion/ } & \text { stay } \\
\text { duration } & \\
\end{array}$ & \multicolumn{3}{|c|}{$\begin{array}{l}\text { Multiple datasets: 1) GPS data from } 252 \text { taxis, } 23 \text { days; } \\
\text { 2) GPS data from } 19 \text { smartphone users, } 12 \text { days; } 3 \text { ) WiFi } \\
\text { data collected with WiFi APs, } 2043 \text { users, } 60 \text { days; 4) } \\
\text { WiFi data collected with WiFi APs, } 804 \text { users, } 370 \text { days }\end{array}$} \\
\hline $\begin{array}{l}\text { Contextual conditional } \\
\text { model } 15\end{array}$ & $\begin{array}{l}\text { next location/ } \\
\text { stay duration }\end{array}$ & $\begin{array}{l}\text { GPS, Blue- } \\
\text { tooth, } \\
\text { WiFi, Call } \\
\text { logs }\end{array}$ & smartphone & $\begin{array}{l}153 \text { users, } 17 \\
\text { months }\end{array}$ \\
\hline $\begin{array}{l}\text { Mobile Data Challenge [1, } \\
16,17,18\end{array}$ & next location & \begin{tabular}{|l|} 
rich smart- \\
phone data
\end{tabular} & smartphone & 80 users, 18 months \\
\hline This work & future location & GPS, WiFi & smartphone & $\begin{array}{l}133 \text { users, } 3-18 \\
\text { months }\end{array}$ \\
\hline
\end{tabular}

ing to efficiently exploit these additional information for location prediction. In the next place prediction task of the Mobile Data Challenge 2012 [1], the best methods relied only on spatio-temporal information to predict future location [16, 17, 18. Closely related to our work, Scellato et al. [14] address the problem of predicting user location at a given time in the near future (e.g., in several hours) instead of the next movement as studied in [1, 15]. However, our work differs from [14] in the following aspects: (1) in addition to learning the time distribution for each place, we also exploit the transition patterns between places by using spatial kernels. (2) We investigate the prediction problem on more complete and dense data. For example, our data has five times more number of places per user than the CenceMe dataset [20] and the percentage of staying time in extracted places is much higher (67\% vs. $15 \%)$. (3) Instead of using time 


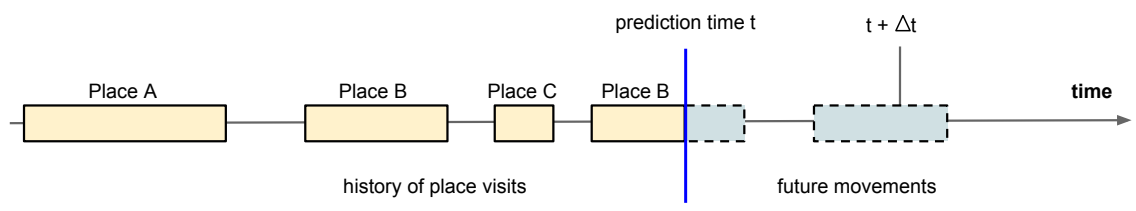

Figure 1: Real-time prediction scenario in which the prediction time $t$ is continuous and the history of place visits increases over time. The fundamental task is to predict the location at time $t+\Delta t$.

series to infer next visiting times of each place then combine predictions with a heuristic method, we propose a probabilistic method which estimates directly the conditional probability of a location at a given time. These probabilities provide generic scores for high-level tasks such as the information retrieval task of predicting the most likely set of places for a time interval.

\section{Prediction framework}

Our framework is built on a high-level representation where location traces are encoded as a history of place visits, for which we only keep visits of significant time periods (e.g., a few minutes) to filter out places that people pass by but did not actually visit. Ideally, one would define places as physical addresses or rooms, which match perfectly the definition of the place in real life. However, this would require an accurate positioning capability for both indoor and outdoor that is beyond what current location tracking systems can provide. For smartphone-based location systems using GPS/WiFi (which are used in this work), the place extraction can output locations that correspond to regions of about 100 -meter radius.

Table 2 illustrates how location traces are stored in our data. Formally, the history is stored as a sequence $H=\left(t_{i}, l_{i}\right)_{i=1 \ldots n}$, where $t_{i}$ is a timestamp that indicates when the user arrived at place $l_{i}, l_{1}$ is the first visited location in the history, and $l_{n}$ is the most recent location of the user (thus it is also the current location). The sequence $\left\{t_{i}\right\}$ must be in increasing order and by construction $l_{i-1} \neq l_{i}$ for all $i$. Furthermore, $l_{i}$ is a positive integer corresponding to a place 
Table 2: Example of location history.

\begin{tabular}{l|l|l} 
Timestamp $\left(t_{i}\right)$ & PlaceID $\left(l_{i}\right)$ & \\
\hline 01-01-2012 12:15:03 & 246 & (a restaurant) \\
01-01-2012 13:35:02 & -1 & (transition) \\
01-01-2012 14:12:15 & 204 & (a friend's place) \\
01-01-2012 17:13:15 & -1 & \\
01-01-2012 17:40:13 & 18 & (home) \\
01-01-2012 20:11:10 & -2 & (phone off) \\
01-01-2012 22:17:15 & 18 & \\
$02-01-2012 ~ 08: 15: 12$ & -1 & \\
02-01-2012 08:30:12 & 376 & (office) \\
02-01-2012 12:30:16 & 275 & (another restaurant) \\
02-01-2012 13:25:56 & 376 & $\ldots$
\end{tabular}

ID, but it can be negative in some special cases:

$\diamond T R A N S=-1$ : the location is unknown or not a significant place. This happens when the user is on the move (i.e., he briefly passes by many non-significant places in the trajectory).

$\diamond O F F=-2$ : the phone or the sensing module are off.

Prediction problem formulation. We are interested in predicting user location at a specific time in the near future. This can be formalized as follows: At time $t$, we want to predict user location at time $t+\Delta t$, given his history of place visits up to time $t$, denoted by $\mathcal{H}_{t}$. The history of visits $\mathcal{H}_{t}$ can be viewed as a training dataset from which we can extract repetitive mobility patterns such as the fact that the user arrives at work around the same time every working day. The time interval $\Delta t$ is the look-ahead time of the prediction, which varies from 5 minutes to 24 hours in our analysis. Intuitively, predicting the near future (for example, the next hour) is easier than predicting user location a long time ahead (for example, in 3 hours). Figure 1 illustrates the prediction setting, where blocks represent place visits and the horizontal axis corresponds to time.

Recall that locations are encoded by abstract place IDs instead of geo- 
location, so that the set of possible outputs of the prediction algorithm is the set of place IDs that the user had visited up to the prediction time (including the TRANS code). Note that the ground truth location may not belong to the considered output set when people visit new places, but we do not introduce a 175 special code "new place" since it corresponds to a small fraction of the data. Based on our collected location traces, we estimate that the average probability of being in a new place at a random time within the next 24 hours is $p=0.03$.

As a simulation of a real-life scenario in which the application makes predictions on the fly, we consider the prediction time $t$ to be continuous. For a single user data, there are multiple training sets which correspond to different values of $t$. Since the training set size increases with $t$, we could expect that the predictability of user movements improves over time.

\section{Location data}

Our experiments were performed on the data from the Lausanne Data Collection Campaign (LDCC) which was ran from October 2009 to the end of March 2011 in Switzerland [21. About 180 volunteer users around Lake Léman participated in the campaign. LDCC participants were asked to carry Nokia N95 phones with recording software running in the background. Thanks to the dynamic sampling technique using a state machine approach, the phone recorded data continuously on a $24 / 7$ basis with the only restriction of having to charge the phone once a day.

Location sensing. The raw location traces were collected by combining GPS and WiFi readings. Since GPS is a power-hungry sensor, the recording software only activates GPS (one reading per 10 seconds) when the phone is detected to be moving. As a complement to GPS data, WiFi readings are made to track user location indoors. The recording software estimates the position of each observed WiFi Access Point (AP) based on GPS readings that are close to AP reading (time difference of less than 90 seconds). 


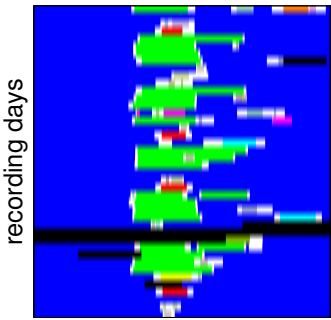

time of day $(0-24)$

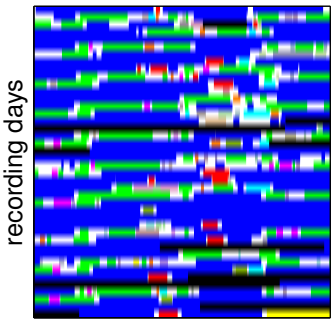

time of day $(0-24)$

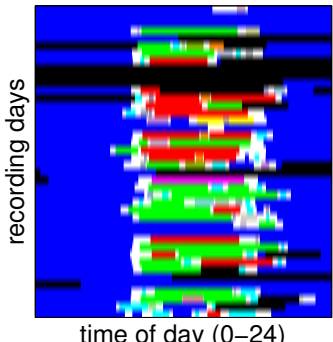

time of day $(0-24)$

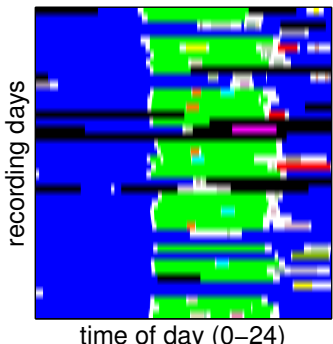

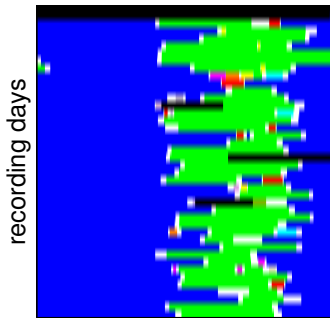

time of day $(0-24)$

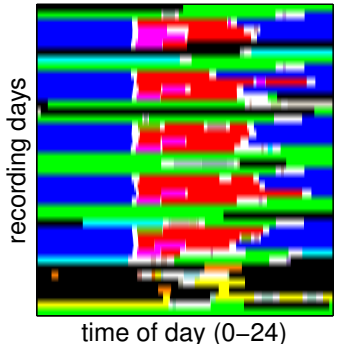

Figure 2: Sample of location data from 6 different users. Rows correspond to days. Black represents missing data, white represents transition state, and other colors correspond to different places that the user visited. Note that places are user-dependent, and that blue corresponds to the user's home.

Extracting the history of place visits. The raw location data points were transformed into a high level representation based on a two-step process proposed by Zheng et al. 22. The details of our implementation can be found in our earlier work 23]. In this work, a location trace is first segmented into transitions and stay points, the minimum time of stay points was set to 10 minutes. Then the set of stay points are clustered into stay regions of 100-meters radius using a grid clustering algorithm. The set of extracted stay regions are used to define places that the user visited. Places are extracted for each user independently of the data of other users.

Data filtering and Statistics. To investigate the prediction task, we filtered out outliers, these were users whose recorded location traces were very incomplete due to technical issues. This filtering step is necessary to avoid biased estimates of prediction performances. At the end, there were $\mathrm{N}=133$ users 


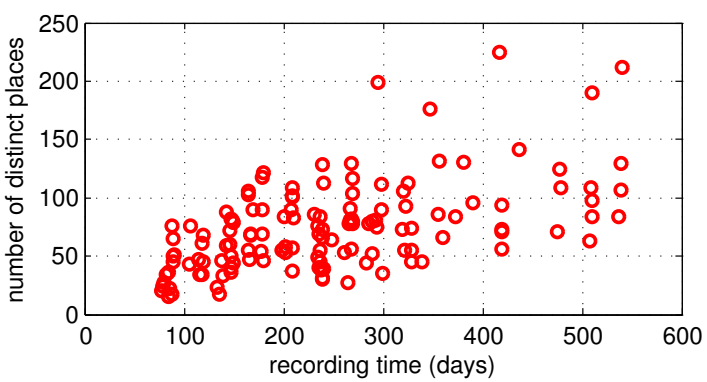

Figure 3: Scatter plot of recording time and number of distinct places, each circle represents a single user.

having location traces satisfying three constraints: a) $30 \%$ of their days with location data; b) recording time of at least 90 days; c) a fraction of missing location data and transitions of less than $70 \%$. Samples of the data are illustrated in Figure 2, The recording time of location traces are plotted in Figure 3, which shows a correlation between the recording time and the number of places that people had visited. On average, each user had visited 75.8 places during the recording period. People were detected to stay $67 \%$ of the time, moving $5 \%$ of the time, while the amount of missing data were $28 \%$.

\section{How difficult is the prediction task? Baseline performance}

In this section, we establish baseline performance for our prediction task based on two basic observations from the mobility data. First, while people usually visit a large number of places in everyday life, their location traces are dominated by a small number of significant places [23]. This observation leads to our first baseline model called MostPopular which predicts future location based on the popularity of visited places. The second observation is that people tend to stay some time in each place, instead of continuously jumping from one place to another. Therefore, a method predicting that the user will be at the same place, called SamePlace, for the near future will have a high accuracy. These two methods are described below:

MostPopular method: This method predicts the future location at time 
$t+\Delta t$ to be the most popular place in the observed history regardless of the context. The most popular place is defined as the place with the largest stay time up to the prediction time $t$. Note that the most popular place can vary over time $t$ (especially at the beginning of the location trace), but it usually converges to the home location after a few days of data collection.

SamePlace method: This method predicts the future location at time $t+\Delta t$ to be the same place as the location at time $t$ regardless of $\Delta t$. Note that if the current location is not available (due to missing data) then this method is unable to predict the future location.

The evaluation was done as follows. First, we generated a set of prediction times $t$ for each location trace, one timestamp every 5 minutes. Then for each $\Delta t$ of interest, we predict the future location at time $t+\Delta t$ with several methods. Finally, the accuracy of a method is the fraction of correct predictions over the total number of predictions. Figure 4 shows the baseline accuracies of predicting user location in the next 3 hours (i.e., $\Delta t=3$ hours) for each user. Looking at the overall accuracies in Figure 4(a), we see that the two baseline methods are competitive, reaching an accuracy of about 0.65 . While these baseline results are relatively high, they are biased by the night periods in which people generally sleep at home so that the prediction is accurate. The effect of time on prediction is clearly highlighted in Figure $4(\mathrm{~b}, \mathrm{c})$ which illustrates the prediction accuracy for daytime (6am-6pm) and night time (6pm-6am) separately. As expected, the prediction accuracy for night time is very high (around 0.81) even with very simple methods. For daytime, the baseline accuracy drops to 0.52 with SamePlace method being more accurate than MostPopular method. While the prediction task is much more challenging for daytime, this is the period of the day in which users are more active and predictions are probably more useful.

Up to now, we only consider a fixed look-ahead time $(\Delta t)$ of 3-hours. While the performance of the MostPopular method is not affected by $\Delta t$, the performance of SamePlace method depends significantly on this variable. Figure 5 reports prediction accuracy as a function of $\Delta t$ in log-scale, showing that the probability of being at the same place is very high for small $\Delta t$, and decreases as 


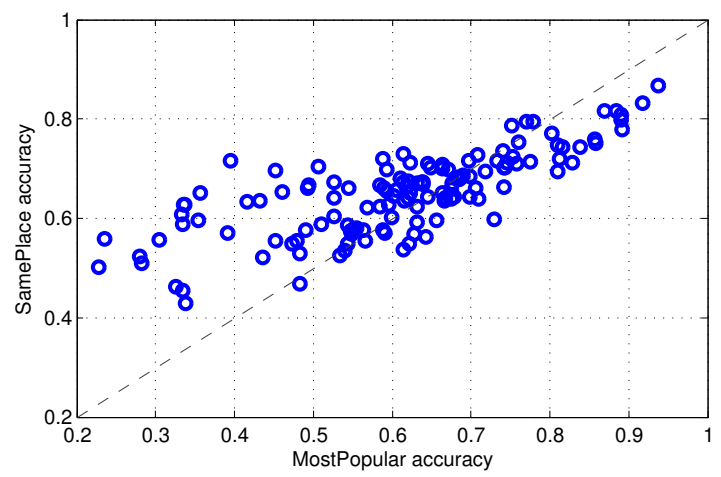

(a) overall accuracy

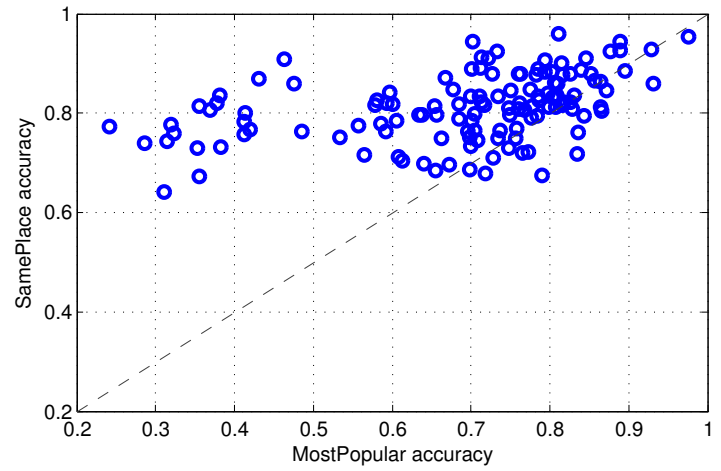

(b) night prediction-time $t$ (6pm-6am)

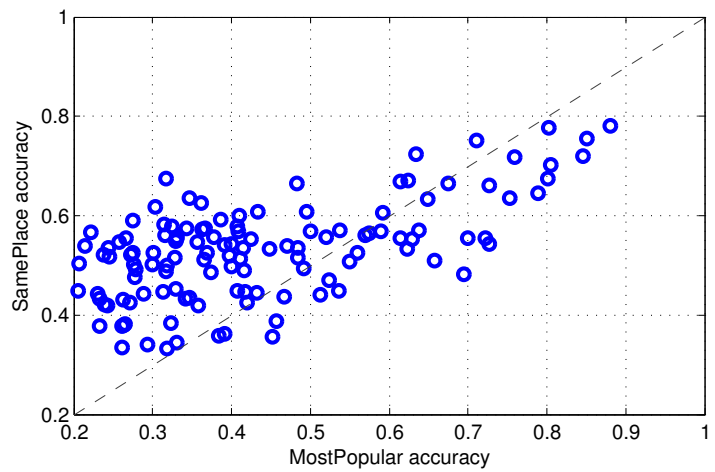

(c) day prediction-time $t(6 \mathrm{am}-6 \mathrm{pm})$

Figure 4: Accuracies of predicting location in the next 3 hours with two baseline methods ( $\Delta t=3$ hours). Each data point corresponds to a user. 


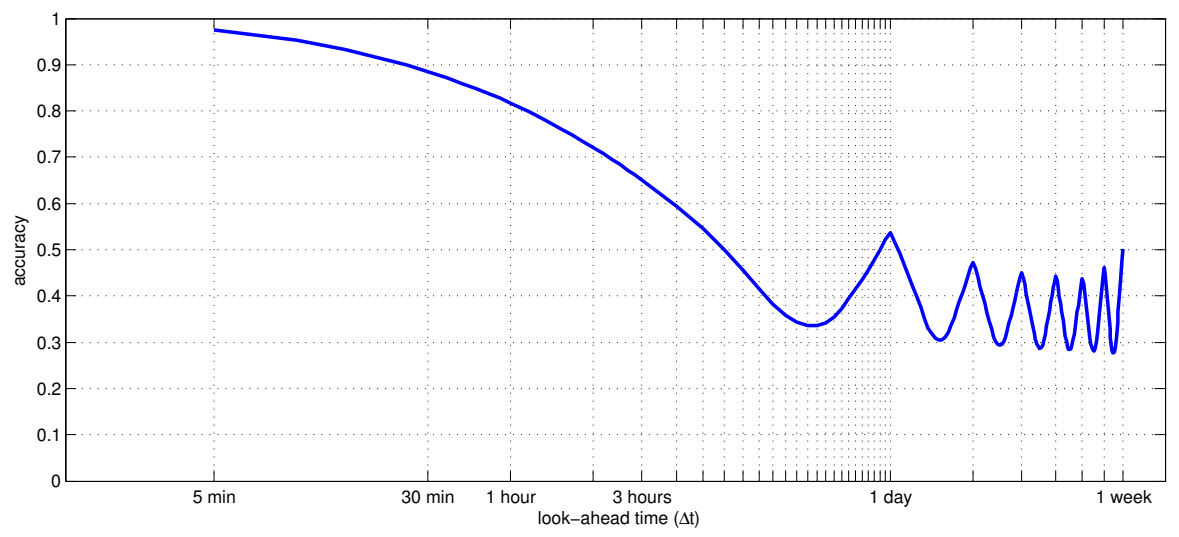

Figure 5: Accuracy of the SamePlace baseline on the LDCC data.

$\Delta t$ increases from minutes to hours. However, the curve has peaks at multiples of 24-hours, reflecting the fact that there is a high chance that the user will be found at the same place at the same time of the following day, the day after, etc. The accuracy of SamePlace model for predicting location in the next 24-hours is 0.54 . This observation suggests that the combination of spatial context (current place) and temporal context (current time) is relevant for predicting future locations, even for large $\Delta t$. In the next section, we will show how to exploit spatial and temporal context in a prediction model based on a combination of a naive Bayes assumption and a kernel density estimation method (KDE).

\section{Our approach: spatio-temporal probabilistic model}

There are several factors that explain why a person is located at a specific place and a given time, including regular routines (such as home-work), needs 275 (such as lunch time), and social relations (such as hanging out with friends). While these factors are highly personal and cannot be entirely integrated in an automatic sensing framework, some of them can be captured using smartphone sensors and statistical methods. We focus on temporal and spatial information and learn the dependencies between these contextual variables and the future location of the user. Temporal context captures regular mobility patterns from 
the weekly calendar, such as being at a particular place at a given time of the day and day of the week. Furthermore, the spatial information is helpful when we aim at exploiting the dynamics of movement, such as going to one specific place after visiting a given place. In the remainder of this section, we first describe how to incorporate different contextual variables in a single probabilistic model. Then we show how hyper parameters are determined using a calibration dataset. Finally, we evaluate the spatio-temporal model and compare it to the baseline methods.

\subsection{Probabilistic framework}

Let $\mathbf{c}$ be all contextual information for the prediction of location at time $t+$ $\Delta t$. Note that the context $\mathbf{c}$ depends on the prediction time $t$ and the look-ahead time $\Delta t$, but we drop the time components to simplify the presentation. To ease the model, we assume that the context information is represented as a fixed length vector where $c_{f}$ denotes the $f^{t h}$ element. To compute the conditional probability of a location $l$ given the context $\mathbf{c}$, we use Bayes' theorem:

$$
P(l \mid \mathbf{c})=\frac{P(l) P(\mathbf{c} \mid l)}{P(\mathbf{c})} \propto P(l) \prod_{f} P\left(c_{f} \mid l\right)
$$

in which the elements of $\mathbf{c}$ are assumed to be independent given the future location $l$. By this formulation, the conditional probability can be factorized into $P\left(c_{f} \mid l\right)$, which are probabilities that a specific context occurs when a user is in place $l$. Compared to the original conditional probability, the elementary probability is "easier" to estimate as it involves less random variables. Our estimation method which is based on KDE is described below.

The probability $P\left(c_{f} \mid l\right)$ can be estimated from the mobility history by sampling data from visits of place $l$. Note that $c_{f}$ can be discrete (e.g., the day of the week) or continuous (e.g., the time of the day) in our framework. One can use a parametric approach, which assumes that the $c_{f}$ random variable follows a certain distribution (such as a Gaussian mixture) whose parameters need to be estimated from the data. As the sampled dataset evolves from 
"sparse" to "dense" (if the place $l$ is frequently visited), this approach requires that we adapt the hypothesis distribution to the density of the data: we should not learn a complex distribution from only a few samples nor to use an oversimplified distribution when the data is dense. Kernel density estimation approach is advantageous in this context, as this non-parametric approach does not require any hypothesis distribution. Intuitively, for a given independent and identically distributed sample drawn, KDE estimates the unknown probability density function by placing a little bump on each training point and summing them. The bump is defined by a smoothing kernel, which takes the "distance" of two data points as input. In this way, the estimated density is high at the area which is close to many data points, and the density function can have any shape depending on the data and the kernel. Note that for discrete variable, we can use the Kronecker delta as a distance measure instead of the Euclidean distance which is used for continuous variables.

As discussed above, the training data is generated from the history of place visits. In practice, we use uniform sampling in which data points are generated every five minutes. The history of visits is then transformed to a pair of a contextual matrix $\mathbf{C}$ and a corresponding location vector $\mathbf{L}$,

$$
\mathbf{C}=\left[\begin{array}{lll}
\bar{c}_{11} & . . & \bar{c}_{1 F} \\
. . & . . & . . \\
\bar{c}_{m 1} & . . & \bar{c}_{m F}
\end{array}\right] ; \mathbf{L}=\left[\begin{array}{c}
\bar{l}_{1} \\
. . \\
\bar{l}_{m}
\end{array}\right]
$$

where $m$ is the number of generated data points (e.g., one data point every 5 minutes), $F$ is the number of extracted contextual information, $\bar{c}_{i f}$ denotes the $f^{t h}$ element of the contextual vector computed for the $i^{\text {th }}$ data point, and $\bar{l}_{i}$ denotes the user location corresponding to $i^{t h}$ data point. The probability $P\left(c_{f} \mid l\right)$ can be estimated as follows:

$$
P\left(c_{f} \mid l\right)=\frac{1}{J(l)} \sum_{i \in J(l)} K_{f}\left(c_{f}, \bar{c}_{i f}\right),
$$

where $J(l)=\left\{i \mid \bar{l}_{i}=l\right\}$ is the set of indices of data points generated from visits of place $l$, and $K_{f}(.,$.$) is a kernel defined on pairs of f^{t h}$ contextual variables 
that will be detailed in the next subsections. The size of the $\mathbf{C}$ and $\mathbf{L}$ matrix spatial context at time $t$ is the sequence of user locations at $t-5 \mathrm{~min}, t-15 \mathrm{~min}$ $t-30 \mathrm{~min}, t-1 \mathrm{~h}, t-2 \mathrm{~h}, t-4 \mathrm{~h}, t-8 \mathrm{~h}, t-24 \mathrm{~h}$, represented by a sequence of 8 place IDs. Note that user location might be unavailable for some timestamps, 
Table 3: List of kernels for various contextual information. The $u \ominus v$ denotes the time difference between two times of the day $u$ and $v$. For temporal information, the time unit of variables $u$ and $v$ is day.

$\left.\begin{array}{|l|l|l|l|}\hline \text { kernel code } & \text { contextual information } c_{f} & \text { kernel type } & \text { parameters } \\ \hline \text { TOD } & \text { time-of-day } & K(u, v)=\mathcal{N}\left(u \ominus v ; 0, \sigma^{2}\right) & \sigma \in R^{+} \\ \hline \text { DOW } & c_{f} \in(00: 00,23: 59) & K(u, u)=1-\lambda, K(u, v)=\frac{\lambda}{6} & \lambda \in(0,1) \\ \hline \text { WE } & \text { day-of-week } & \forall v \neq u\end{array}\right]$

which is represented by the special code $O F F$ discussed in Section 3 .

We used four temporal kernels and one spatial kernel:

- TOD: a continuous kernel between times of the day, which is defined as a normal distribution over time-differences between the two timestamps with zero mean and variance $\sigma^{2}$.

- DOW: a discrete kernel between days of the week, parameterized by $\lambda$ between 0 and 1 .

- WE: a discrete kernel for day categories (weekend vs. weekday), parameterized by $\lambda$ between 0 and 1 .

- TimeD: a discrete kernel for the time difference between two timestamps $u$ and $v$, parameterized by $\tau$ and $\lambda$. The kernel outputs two possible values depending on whether the time difference exceeds the threshold $\tau$.

- Place: a discrete kernel between spatial contexts. The kernel between two location sequences can be factorized as a product of elementary kernels, computed for each timestamp of the two sequences. The elementary kernel between places is a discrete kernel parameterized by a single parameter $\lambda$. Note that the normalization constant $Z$ can be omitted in the computation 


$$
\text { of } P(l \mid \mathbf{c}(t, \Delta t)) \text {. }
$$

Besides the spatial kernel listed above, we also explored more sophisticated kernels based on the distances, note however that this kernel can not be applied to abstract location such as WiFi fingerprint. A straightforward solution is to 375 define a normal kernel over the geo-distances among places. We also developed a finer distance kernel by extracting a feature vector of each place (e.g., average staying time, visit frequency), and then using a multivariate normal distribution with diagonal covariance matrix to define the kernel between places. Unfortunately, these spatial kernels were not better than the discrete place kernel above and did not help to improve the performance of the whole system. For this reason, we did not include the results with these spatial kernels in the analysis.

\subsection{Kernel bandwidth optimization}

Kernel bandwidths are hyper-parameters which define the smoothness of the estimated density functions. The larger the bandwidth, the smoother the density curve.

The set of parameters in Table 3 can be optimized automatically based on a training dataset. Each parameter is optimized sequentially to maximize the conditional likelihood on the training set using a heuristic search. Starting with an empty set of kernels (i.e., only use the prior), we iteratively add one kernel type and find the best parameters for that kernel on the sample data of a few users. Among an exponential number for kernel ordering, we chose to start with temporal kernels and end with the spatial kernel as in Table 3 . At the first iteration, we optimize the parameter $\sigma$ of the kernel $T O D$ in the model with only 1 kernel. At the second iteration, the model has two kernels, TOD and process iterates until the parameter $\lambda$ of the last kernel (Place) is optimized. The order of kernel parameters to be optimized can, theoretically, affect the system since the optimization problem is not convex. Among an exponential number of possible kernel orders, we did some tests and did not find any significant 400 
We also divide the space of look-ahead time $\Delta t$ into multiple intervals, and then optimize the kernel parameter for each interval separately. The intuition behind this technique is that the importance of each kernel may vary depending on $\Delta t$. For example, spatial information can be important for predicting the next hour, but it is not very helpful for predicting location in 10 hours. We implement this technique with 3 time intervals for $\Delta t$ : from 0 to 1 hour, from 1 to 3 hours, and more than 3 hours.

\subsection{Evaluation of Spatio-Temporal model}

In this section, we first study the contribution of each kernel to the overall accuracy of our model, and then we compare the method with the baseline results. To make the results generalizable, we always perform cross testing. In other words, to evaluate the prediction performance on a given user, we always use the set of parameters optimized on a training set that does not include that user. In general, the training data contains only 3 or 4 users, which is enough to find a good set of parameters. A few experiments with larger number of users show that the performance is not improved significantly.

Starting with a model with the TOD kernel only, we sequentially add more kernels to the model and study how the accuracy is improved. Figure 6 shows the performance of our method with an increasing number of kernels. As can be seen, the combination of the two kernels TOD and DOW results in a competitive performance for predicting the next 3 hours compared to the model that uses all four of the temporal kernels. For larger $\Delta t$, the WE and TimeDiscrete kernels are found to be useful in improving prediction accuracy.

A considerable improvement can be observed after adding spatial information to the model, especially for the prediction of the next few hours. For example, the accuracy of predicting location in 1 hour increases from 0.72 to 0.83 by adding the Place kernel.

Besides the two simplistic baseline versions, namely MostPopular and SamePlace, we also compare our proposed method with spatial-temporal Markov model [1], called MarkovCDF, which is considered to be among the most accu- 


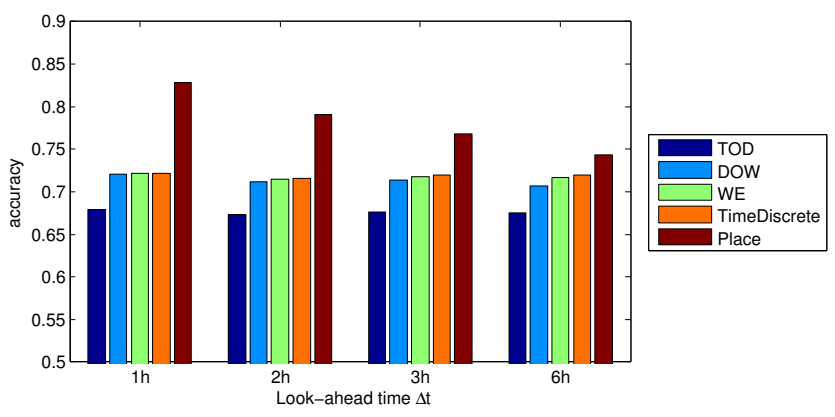

Figure 6: Accuracy of the spatio-temporal model with increasing number of kernels. The legend should be read as accumulated. Blue corresponds to the model with the TOD kernel only. Dark red corresponds to the model with all the five kernels.

rate prediction methods for this class of prediction problems [14]. The method sequentially predicts the next destination and the arrival time by combining the transition probability between places and the distribution of visit and transition durations. The result of a 3rd order model is slightly worse than the 2nd order model, we only report the results of the 1st and 2nd order Markov model, noted MarkovCDF(1) and MarkovCDF(2) respectively.

Figure 7 shows comparative results of our kernel method, called SpatioTemporal, and the baseline methods. The Temporal method corresponds to our method with only temporal kernels. As can be seen, both the Temporal and the Spatio-Temporal models significantly outperform both the simplistic baseline MostPopular and the more sophisticated baseline MarkovCDF, for all look-ahead time $\Delta t$. These results can be explained by two key advantages of the proposed method over the MarkovCDF. First, while the MarkovCDF method only uses the history data points with the exact match of current context, our method defines soft-matching scores between context vectors via kernel function, allowing us to exploit the historical data more efficiently. Second, the MarkovCDF needs to sequentially fill the location trace from $t$ to $t+\Delta t$ by predicting next location and arrival time. In this greedy approach, a single prediction error will be propagated to subsequent predictions. Our approach instead provides a direct estimate of the conditional probability of a location at 


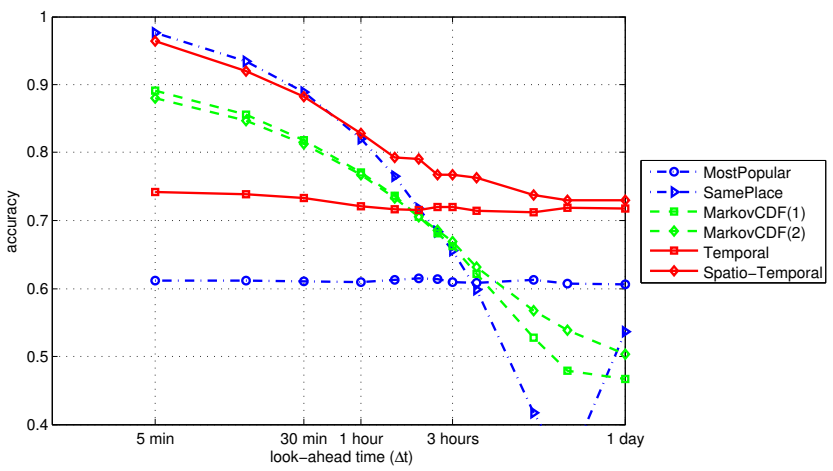

Figure 7: Comparing kernel-based models with baselines on the WiFi dataset. The Temporal curve corresponds to the model that includes the kernels on temporal information (TOD,DOW,WE,TimeDiscrete). The spatio-temporal model corresponds to the model with kernels on all computed features.

time $t+\Delta t$, thus avoiding the propagation of errors in greedy methods [11, 14].

Finally, we see that the performance of the spatio-temporal option is generally better than the one of SamePlace method. However, the SamePlace method is still better than the Spatio-Temporal model for the look-ahead time of less than 1 hour. This reflects the fact that our model does not completely capture the same place probability distribution. This observation suggests that we could improve the spatio-temporal model by combining it with the SamePlace method.

\section{Combining Spatio-Temporal Model with SamePlace model}

To combine SamePlace with our kernel method, we first introduce a probabilistic model for the SamePlace method, then employ a convex combination of probabilities as follows:

$$
P(l \mid \mathbf{c}, t, \Delta t)=\alpha_{t, \Delta t} P_{s t}(l \mid \mathbf{c})+\left(1-\alpha_{t, \Delta t}\right) P_{s p}(l \mid t, \Delta t),
$$

where $P_{s t}($.$) is the probability estimated by the Spatio-Temporal model and$ $P_{s p}(l \mid t, \Delta t)$ is the probability given by the probabilistic SamePlace model which we will detail in the next paragraph. By combining the Spatio-Temporal model 
with the SamePlace model, we expect the two models to complement each other in different situations. Importantly, the convex combination of two distributions results in a probabilistic distribution without any additional adaptation. The model remains in a probabilistic framework.

\subsection{Probabilistic SamePlace model}

Our goal is to model the probability that the user will be at the same place for a given $\Delta t: P_{s p}(l \mid t, \Delta t) \triangleq P(l(t+\Delta t)=l(t))$ where $l(t)$ denotes the location at time $t$. Without any contextual information, this probability coincides with the curve in Figure 5 if there is no missing data. However, the probability of being at the same place after $\Delta t$ depends on many factors. We implemented a simple method which uses the time of day as a conditional variable. The intuition is that the probability of being at the same place after e.g., 3 hours would be high for night timeslots (e.g., 3am) while it would be low for the early morning (e.g. 7am). At the end, for each hour of the day $h$ and each $\Delta t$, we have an estimate of $\hat{p}(h, \Delta t)$ for the probability of being at the same place after a time interval $\Delta t$ given the current hour $h$. Finally, the probability of being at a place $l$ at time $t+\Delta t$ with the probabilistic same-place model is defined as follows:

$$
P_{s p}(l \mid t, \Delta t)= \begin{cases}\hat{p}(h(t), \Delta t) & \text { if } l=l(t) \text { and } l(t) \neq O F F \\ \frac{1-\hat{p}(h(t), \Delta t)}{M-1} & \text { if } l \neq l(t) \text { and } l(t) \neq O F F \\ \frac{1}{M} & \text { if } l(t) \neq O F F\end{cases}
$$

where $h(t)$ is the hour at the prediction time $t, M$ is the number of visited places in the past, and $O F F=-2$ is the special code for missing data.

The probabilistic same-place model can be learned for each individual or for a population. In our implementation, we use data from all users to estimate a general model for the LDCC population. This general model performs well in aggregate, but can be inaccurate for outliers (e.g., a person that works a night shift). We leave as future work the implementation of a personalized probabilistic same-place model. 


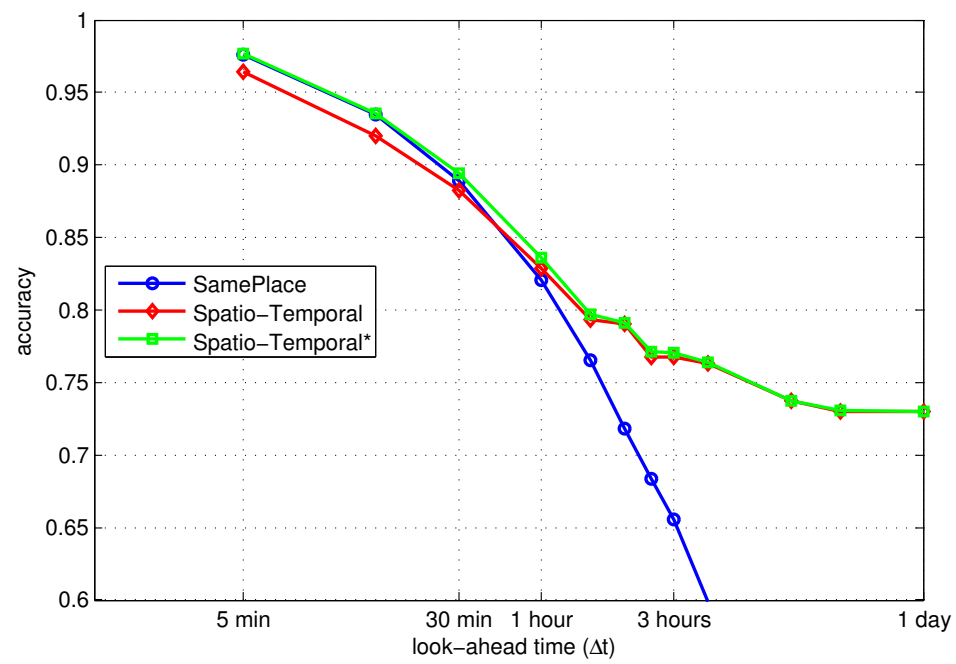

Figure 8: Overall accuracies of the combined model Spatio-Temporal*, compared to the SamePlace baseline and the Spatio-Temporal model.

For the combination weight in Eq. (4), we also found that the optimal value for $\alpha$ varies in function of the time of day and $\Delta t$. To maximize the performance of the combination model, we optimize $\alpha_{t, \Delta t}$ for each combination of time of day and $\Delta t$. The time of the day was divided uniformly into 12 intervals, and the duration of $\Delta t$ is divided into 4 intervals: 0-30 minutes, 30 minutes - 1 hour, 1 - 2 hours, and more than 2 hours. Similarly to kernel parameters, the values of $\alpha$ for each case is optimized on the training data.

\subsection{Combination results}

Figure 8 shows the results obtained with the proposed combined model, noted as Spatio-Temporal*. As can be seen, the combined model improves the accuracies of Spatio-Temporal models for $\Delta t$ less than 1 hour, and slightly outperforms the SamePlace baseline in term of accuracies. It is also interesting to see that combined method provides some improvements for $\Delta t$ larger than 1 hour, although the difference is small. This is not a surprise since the sameplace probability is relatively low for $\Delta t>3 h$, and so becomes less useful for the prediction of future location. 


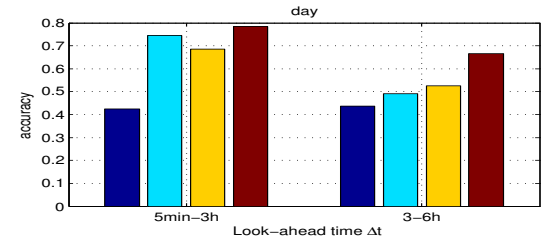

(a) daytime

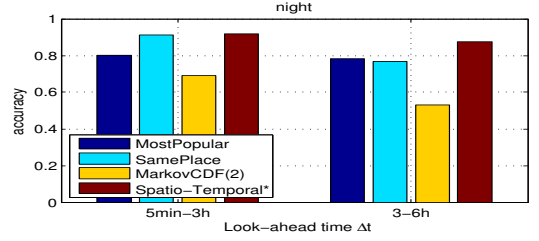

(b) night period

Figure 9: Comparison of prediction accuracy of prediction methods at two periods of the day: 6am-6pm (daytime), 6pm-6am (nighttime), and various look-ahead times $\Delta t$.

Figure 9 illustrates the effect of the prediction time on the accuracies of the prediction methods. As human mobility is highly predictable for the night period, we have good accuracies even with baseline methods that do not exploit any contextual information (also see Figure 44). The prediction accuracies for night periods can be improved by exploiting contextual information (in the spatio-temporal and combined models). However, these improvements are modest compared to the larger improvements obtained for daytime prediction, in which human mobility is more complex.

Considering location prediction in the next three hours, we see that the accuracies of the baseline SamePlace model is very high (for daytime prediction the accuracy is 0.74 ), and the prediction accuracies cannot be improved much by the contextual model. Any improvement in accuracies probably comes from the low fraction of time in which people move within three hours. These periods, however, are interesting from an applicative view point. When $\Delta t$ increases, the differences between the combined model and the SamePlace baseline are more significant since the performance of this baseline degrades quickly. Our methods perform better than baseline methods in many situations.

The comparative analysis in this section shows that our methods perform better overall, in many situations, than the baseline methods. While the SamePlace model can be very accurate, the prediction is not interesting in practice. This finding indicates that the accuracy measure is not enough for the evaluation of predictors. In the next section, we consider a more applicative prediction 
task with an alternative evaluation measure. an approximate order. We explore this task here.

\subsection{Prediction task and evaluation measures}

In the previous sections, we studied the task of predicting a unique user location at a given time in the future. This task can be generalized to the case of predicting several places that a user will visit within a time interval in the near future. The task is formalized as follows: At time $t$, predict the list of places that the user will visit in the time interval $\left(t+\Delta t_{1}, t+\Delta t_{2}\right)$ given the history of place visits up to time t. This task can be viewed as an information retrieval task if we consider the current context as the query, the list of places 25. The predictor gives a score for each place, which is used to rank the set of places. We use standard information retrieval measures to evaluate the sorted list of places produced by the predictor. Let $\mathrm{N}$ be the number of places in the response; the evaluation measures are computed as follows:

535

- precision at $\mathrm{N}$ : the fraction of the top-N places that are actually visited in the time interval $\left(t+\Delta t_{1}, t+\Delta t_{2}\right)$.

- recall at $\mathrm{N}$ : the fraction of visited places that belong to the list of top- $\mathrm{N}$ places in the response.

- F-score at $\mathrm{N}$ : the harmonic mean of precision at $\mathrm{N}$ and recall at $\mathrm{N}$ :

$$
F=2 \cdot \frac{\text { precision } \cdot \text { recall }}{\text { precision }+ \text { recall }}
$$




\subsection{Adapting predictors to the new task}

Our proposed model can be easily adapted to perform this task by aggregating prediction results from multiple predictions with various $\Delta t \in\left(\Delta t_{1}, \Delta t_{2}\right)$. We define

$$
\operatorname{score}\left(l \mid t, \Delta t_{1}, \Delta t_{2}\right)=\sum_{\Delta t \in\left(\Delta t_{1}, \Delta t_{2}\right)} P(l \mid t, \Delta t),
$$
case of large proportion of missing data (higher than 30\%) in the prediction time interval $\left(t+\Delta t_{1}, t+\Delta t_{2}\right)$, the predicted results are not included in the evaluation due to incompleteness of ground truth. 


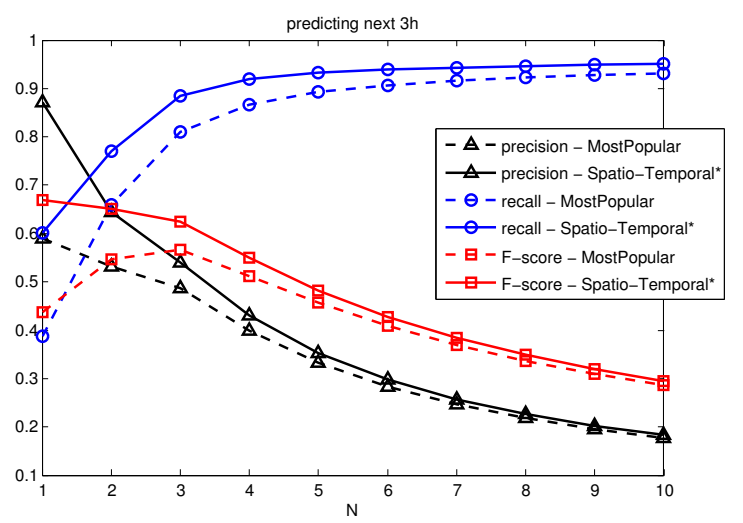

Figure 10: Precision, recall, and F-score values for predicting the set of $\mathrm{N}$ most likely locations for the next 3 hours.

\subsection{Results on prediction location for a time interval}

Figure 10 shows experimental results for the task of predicting user location for the next 3 hours (that is, $\Delta t_{1}=0$ and $\Delta t_{2}=3 h$ ). Recall that we consider four daily prediction times (at 8:00, 11:00, 14:00, and 17:00), which correspond to the four prediction time intervals: 8:00-11:00, 11:00-14:00, 14:00-17:00, and 17:00-20:00. Two models are compared: the non-contextual approach with the MostPopular model (Section 5), and the contextual approach with the SpatioTemporal* model from Section 7. We compare the performance of the two models by using the three aforementioned evaluation measures with $N$ ranging from 1 to 10 . As can be seen, the Spatio-Temporal* model systematically outperforms the baseline model for all evaluation measures and all values of $N$. As people generally do not visit too many places within 3 hours, the precision value drops quickly as $N$ increases. The improvement over the baseline results is also larger for small values of $N$. For example, the absolute improvements in top-1, top-3, and top-5 F-score are $0.23,0.04$, and 0.03 respectively.

To study the effect of look-ahead time in the performance, we shift the prediction time interval by 3 hours $\left(\Delta t_{1}=3 h, \Delta t_{2}=6 h\right)$. Results are shown in Figure 11. Note that the 4 daily prediction time intervals become: 11:00-14:00, 14:00-17:00, 17:00-20:00, and 20:00-23:00. The final results with the second 


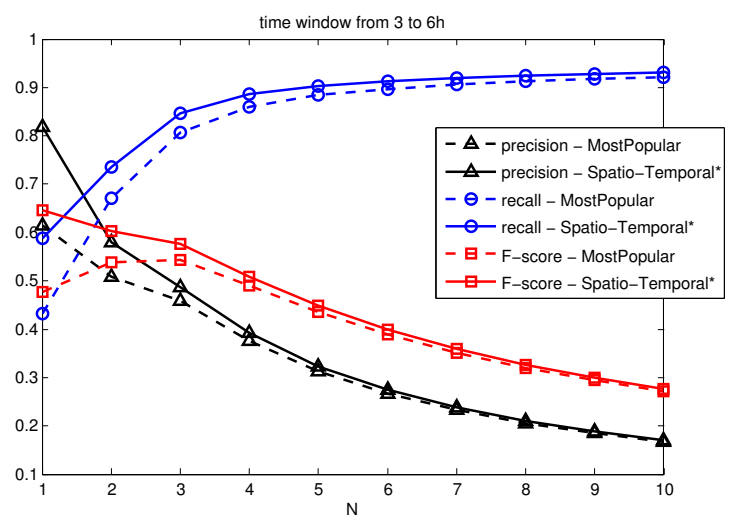

Figure 11: Precision, recall, and F-score values for predicting the set of $\mathrm{N}$ most likely locations for the time window from 3 to 6 hours in the future.

setting follow similar trends that were observed in the first setting, but the absolute values of precision, recall, and F-score are lower. On one hand, the prediction is more difficult because the look-ahead time is increased. On the other hand, the prediction time intervals are shifted towards the evening period, where the human mobility is more predictable. Note also that these two effects reduce the improvement of the Spatio-Temporal* model over the baseline in term of accuracy (see Figure 9), which explains why the improvement in this setting is lower than in the first setting (i.e., predicting the next 3 hours).

It is also relevant to study the prediction performance on different times of the day. We report these results in Figure 12 In term of F-score, the predictability is highest at 14:00 (best $\mathrm{F}$-score $=0.72$ for $\mathrm{N}=1$ ) and lowest at 17:00 (best $\mathrm{F}$-score $=0.66$ for $\mathrm{N}=3$ ) and 11:00 (best $\mathrm{F}$-score $=0.67$ for $\mathrm{N}=2$ ). This reflects the fact that people usually stay in the same place between 14:00 and 17:00 (e.g., working in an office), so that the response for a single place gets the best F-score. At lunch time 11:00-14:00, the best result is obtained for $\mathrm{N}=2$ since people usually go out for lunch, then come back; however, it is not easy to predict exactly where user will go among multiple places. Predictions at 8:00 in the morning (best $\mathrm{F}$-score $=0.7, \mathrm{~N}=3$ ) are generally more accurate than predictions at 17:00 (best $\mathrm{F}$-score $=0.66, \mathrm{~N}=3$ ). While the two prediction time 


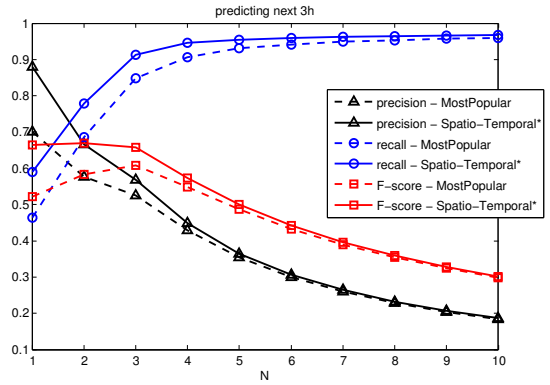

(a) prediction at 8:00

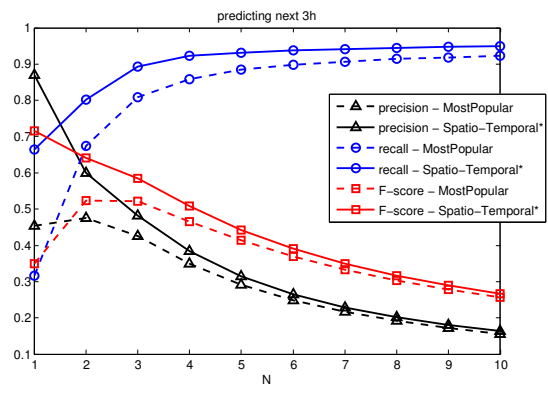

(c) prediction at 14:00

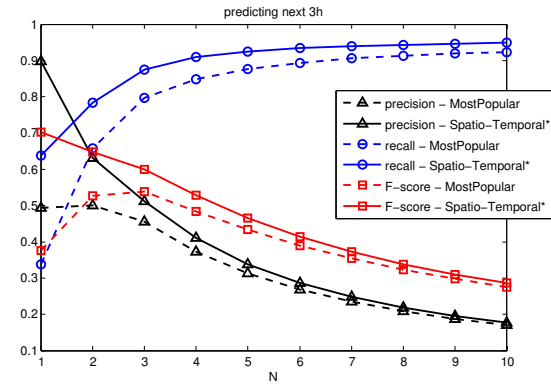

(b) prediction at 11:00

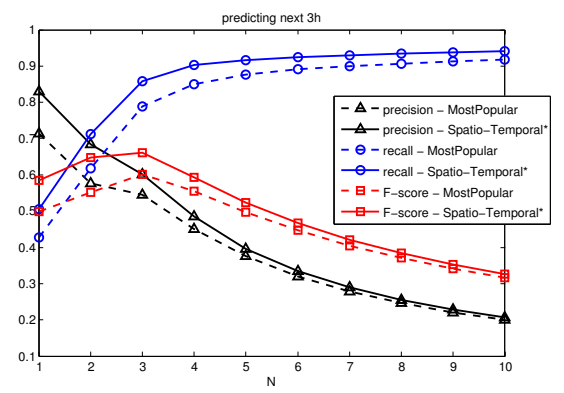

(d) prediction at 17:00

Figure 12: Precision and recall value for predicting the set of $\mathrm{N}$ most likely locations for the next 3 hours at different time of the day.

intervals correspond to commuting transition periods, the morning transition is more predictable than the late-afternoon transition since people can go to different places (e.g., shopping, bars) before returning home. We also observe that the improvement of contextual prediction over non-contextual prediction is highly correlated with the predictability. The contextual information is most helpful for the difficult prediction time interval starting at 11:00.

Finally, we show how predictability varies among users. The scatter plot of top-3 F-scores is presented in Figure 13 which shows that the prediction performance is improved with the Spatio-Temporal* model for most users (above the $45^{\circ}$ line). Moreover, the improvement is large for users having low F-score with the baseline method. Importantly, the variance in performance for the combined method is smaller than the one for the baseline. Finally, the F-scores 


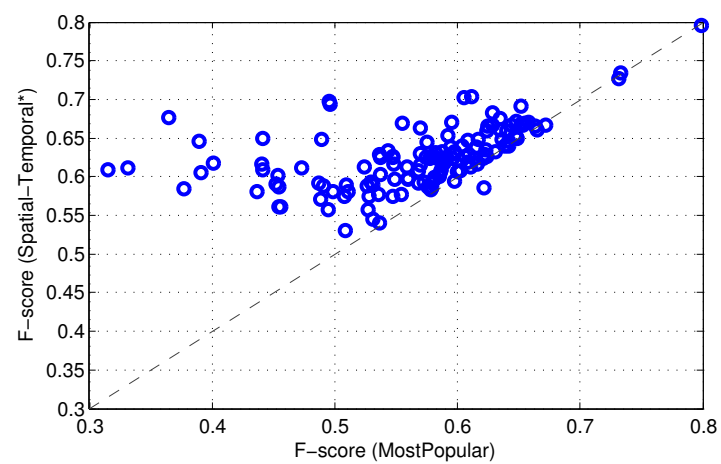

Figure 13: Top-3 F-scores of the prediction of user location in the next 3 hours with MostPopular model and Spatio-Temporal* model. Each circle corresponds to a user in the LDCC data.

seem to follow the normal distribution with mean 0.62 and standard deviation 0.04 .

\section{Conclusion}

We explored the location prediction problem with a new angle by redefining the prediction task. We proposed a probabilistic kernel method for learning the dependency between user location and multivariate contextual variables from sparse data. This is the first study, to our knowledge, that uses the kernel density estimation on multiple temporal and spatial variables for location prediction. To improve the prediction performance, we combined the spatio-temporal model with a baseline model, resulting in a robust probabilistic model which outperforms baseline methods in different situations. Our experiments showed promising results for practical applications of the prediction method, with $84 \%$ accuracy for the location prediction of the next hour, and $77 \%$ accuracy for the next three hours. For the tasks of inferring the most likely set of places for a given interval, our method reaches an accuracy of $93 \%$ with $\mathrm{N}=5$ location candidates.

630

A preliminary version of the method has been implemented on the phone and we did not have any major practical issues. Kernel bandwidths are estimated 
once, offline, and no more parameter estimation is required since the method is non-parametric. This is an advantage compared to the parametric approach where model parameters need to be updated regularly as the train dataset grows. One limitation of the model is that the required memory and computational resources grows linearly with time. In practice, we can define an upper bound on the size of the data where old data points (e.g., less than 3 months) are automatically removed. Other strategies of data elimination which consider the redundancy are also interesting, we will leave this direction for further studies.

As future work, we are interested in the modeling of trajectory which can improve the prediction performance when a user is on the move. Another direction is to integrate social context to the prediction algorithm by using social sensors and/or considering collaborative prediction tasks for multiple users.

\section{Acknowledgment}

Trinh MinhTri Do and Daniel Gatica-Perez acknowledge the support of Nokia Research through the LS-CONTEXT project. Markus Miettinen and Olivier Dousse were with NRC-Lausanne when this research was done. We thank Juha Laurila and Jan Blom for discussions.

\section{References}

650

655

[1] J. Laurila, D. Gatica-Perez, I. Aad, J. Blom, O. Bornet, T. Do, O. Dousse, J. Eberle, M. Miettinen, The mobile data challenge: Big data for mobile computing research, in: Proc. MDC Workshop, 2012.

[2] E. Z. Q. Y. Kaixiang Mo, Ben Tan, Your phone understands you, in: Proc. MDC Workshop, 2012.

[3] G. Chittaranjan, J. Blom, D. Gatica-Perez, Mining large-scale smartphone data for personality studies, Personal and Ubiquitous Computing (2012) $1-18$. 
[4] J. Hightower, S. Consolvo, A. LaMarca, I. Smith, J. Hughes, Learning and recognizing the places we go, in: Ubiquitous Computing, Vol. 3660, Springer Berlin / Heidelberg, 2005, pp. 159-176.

[5] D. Ashbrook, T. Starner, Using GPS to learn significant locations and predict movement across multiple users, Personal Ubiquitous Computing 7 (2003) 275-286.

[6] T. Liu, P. Bahl, I. Chlamtac, Mobility modeling, location tracking, and trajectory prediction in wireless ATM networks, Selected Areas in Communications 16 (6) (1998) 922-936.

[7] R. Bajaj, S. L. Ranaweera, D. P. Agrawal, GPS: location-tracking technology, Computer 35 (4) (2002) 92-94.

[8] C. Song, Z. Qu, N. Blumm, A.-L. Barabsi, Limits of predictability in human mobility, Science 327 (5968) (2010) 1018-1021.

[9] B. Jensen, J. Larsen, K. Jensen, J. Larsen, L. Hansen, Estimating human predictability from mobile sensor data, in: MLSP, 2010, pp. 196-201.

[10] M. Lin, W. Hsu, Z. Lee, Predictability of individuals' mobility with highresolution positioning data, in: Proc. UbiComp, 2012, pp. 381-390.

[11] L. Song, D. Kotz, R. Jain, X. He, Evaluating next-cell predictors with extensive wi-fi mobility data, in: Transactions on Mobile Computing, 2004, pp. 1414-1424.

[12] J. Krumm, E. Horvitz, Predestination: Inferring destinations from partial trajectories, in: Proc. UbiComp, 2006, pp. 243-260.

[13] J. Krumm, A. J. B. Brush, Learning time-based presence probabilities., in: Proc. Pervasive, 2011, pp. 79-96.

[14] S. Scellato, M. Musolesi, C. Mascolo, V. Latora, A. T. Campbell, Nextplace: a spatio-temporal prediction framework for pervasive systems, in: Proc. Pervasive, Berlin, Heidelberg, 2011, pp. 152-169. 
[15] T. M. T. Do, D. Gatica-Perez, Contextual conditional models for smartphone-based human mobility prediction, in: Proc. UbiComp, 2012.

[16] E. K. Vincent Etter, Mohamed Kafsi, Been there, done that: What your mobility traces reveal about your behavior, in: Proc. MDC Workshop, 2012 .

${ }_{690}[17]$ B. P. Jingjing Wang, Periodicity based next place prediction, in: Proc. MDC Workshop, 2012.

[18] H. L. Huiji Gao, Jiliang Tang, Mobile location prediction in spatio-temporal context, in: Proc. MDC Workshop, 2012.

[19] E. Horvitz, J. Krumm, Some help on the way: Opportunistic routing under uncertainty, in: Proc. UbiComp, 2012.

[20] E. Miluzzo, N. Lane, S. Eisenman, A. Campbell, Cenceme-injecting sensing presence into social networking applications, Smart Sensing and Context (2007) 1-28.

[21] N. Kiukkonen, J. Blom, O. Dousse, D. Gatica-Perez, J. Laurila, Towards rich mobile phone datasets: Lausanne data collection campaign, in: Proc. ICPS, Berlin, 2010.

[22] V. W. Zheng, Y. Zheng, X. Xie, Q. Yang, Collaborative location and activity recommendations with gps history data, in: Proc. WWW, ACM, 2010 .

[23] T. M. T. Do, D. Gatica-Perez, The places of our lives: Visiting patterns and automatic labeling from longitudinal smartphone data, Transaction on mobile computing, in press.

[24] A. Peddemors, H. Eertink, I. Niemegeers, Predicting mobility events on personal devices, Pervasive Mob. Comput. 6 (2010) 401-423.

[25] R. Baeza-Yates, B. Ribeiro-Neto, et al., Modern information retrieval, Vol. 463, ACM press New York., 1999. 